\title{
MINIMAL SOLUTIONS OF THE RATIONAL INTERPOLATION PROBLEM
}

\author{
TERESA CORTADELLAS BENÍTEZ, CARLOS D'ANDREA, AND EULÀLIA MONTORO
}

\begin{abstract}
We explore connections between the approach of solving the rational interpolation problem via resolutions of ideals and syzygies, and the standard method provided by the Extended Euclidean Algorithm (EEA). As a consequence, we obtain explicit descriptions for solutions of minimal degrees in terms of the degrees of elements appearing in the EEA. This result allows us to describe the minimal degree in a $\mu$-basis of a polynomial planar parametrization in terms of a critical degree arising in the EEA.
\end{abstract}

\section{INTRODUCTION}

Let $\mathbb{K}$ be a field, $l, n_{1}, \ldots, n_{l}$ positive integers, $n:=n_{1}+\cdots+n_{l}$, and

$$
\left(x_{i}, y_{i, j}\right) \in \mathbb{K}^{2}, \quad \text { for } i=1, \ldots, l, \quad j=0, \ldots, n_{i}-1,
$$

with $x_{i} \neq x_{j}$ if $i \neq j$. An interpolating rational function associated to this data is a function $y(x) \in \mathbb{K}(x)$ satisfying

$$
y^{(j)}\left(x_{i}\right)=y_{i, j}, \quad \text { for } i=1, \ldots, l, \quad j=0, \ldots, n_{i}-1 .
$$

In particular, we are requiring that the rational function $y(x)$ is defined on all the points $x_{i}, i=1, \ldots, l$.

The rational interpolation problem is to describe all the rational functions satisfying (1.2). Note that in principle the well-known interpolating polynomial associated to the data (1.1) is always a solution of $(1.2)$, so this problem is always solvable. Are there more solutions? How many? Can we parametrize them? Is there a minimal or compact solution?

Rational interpolation has been well studied in the last centuries, with references going back to the mid 1800's ([5, 14, 12]). In the last decades, the interest in this problem focused on the more algorithmic and computational aspects, due to the

2010 Mathematics Subject Classification. Primary 41A20; Secondary 13D02, 11 Y50.

Key words and phrases. Rational interpolation; Syzygies; Extended Euclidean Algorithm; minimal degree; $\mu$-basis.

T. Cortadellas is supported by the Spanish research project MTM2016-78881-P; C. D'Andrea and E. Montoro are supported by the Spanish MINECO/FEDER research project MTM 201565361-P. C. D'Andrea is also supported by the "María de Maeztu" Programme for Units of Excellence in R\&D (MDM-2014-0445), and by the European Union's Horizon 2020 research and innovation programme under the Marie Sklodowska-Curie grant agreement No. 675789. 
significant growth of these areas of research and applications: see, for instance, [15, 10, 18, 4, 16] and the references therein. A unified framework, which relates the rational interpolation problem with the Euclidean Algorithm, is presented in [2], and also in the book [17. Section 5.7], where this problem is called rational function reconstruction. There are also explicit closed formulae in terms of the input data that can be derived by applying symmetric operators or subresultants; see [11, 8].

In this paper, we focus on the possible degrees that a solution of 1.2 can have, and detect the minimum of these degrees in optimal time. To be more precise, a degree for a rational function $y(x)=\frac{a(x)}{b(x)}$, with coprime $a(x), b(x) \in \mathbb{K}[x]$, can be defined as

$$
\delta(y(x))=\max \{\operatorname{deg} a(x), \operatorname{deg} b(x)\}
$$

or

$$
\kappa(y(x))=\operatorname{deg} a(x)+\operatorname{deg} b(x) .
$$

Fixing one of these degrees, we say that $\gamma \in \mathbb{N}$ is an admissible degree if there exists an interpolating function of degree $\gamma$. A natural question to ask is which numbers are admissible degrees, and in particular to characterize the smallest admissible degree. A minimal solution of the rational interpolation problem is a solution of minimal degree. Also, it is of interest to parametrize all the admissible rational functions. For instance, for the data

$$
\begin{array}{ll}
x_{1}=0, & y_{1,0}=-2, \\
x_{2}=2, & y_{2,0}=6, \\
x_{3}=-1, & y_{3,0}=-3, \\
x_{3}=-1, & y_{3,1}=3,
\end{array}
$$

it turns out that its $\delta$-minimal degree is 2 and the fractions $\frac{-2-3 \lambda x^{2}}{\frac{-x^{2}}{3}+1-\lambda x}$ define the minimal interpolating functions for any $\lambda \in \mathbb{K}, \lambda \neq-\frac{1}{6},-\frac{2}{3}$. Moreover, the interpolating functions $y(x)=\frac{a(x)}{b(x)}$ with $\delta(y(x))=\delta>2$ can be parametrized as

$$
y(x)=\frac{\left(\lambda_{0}+\cdots+\lambda_{\delta-2} x^{\delta-2}\right) r_{2}(x)+\left(\lambda_{0}^{\prime}+\cdots+\lambda_{\delta-3}^{\prime} x^{\delta-3}+x^{\delta-2}\right) r_{3}(x)}{\left(\lambda_{0}+\cdots+\lambda_{\delta-2} x^{\delta-2}\right) s_{2}(x)+\left(\lambda_{0}^{\prime}+\cdots+\lambda_{\delta-3}^{\prime} x^{\delta-3}+x^{\delta-2}\right) s_{3}(x)}
$$

for suitable polynomials $r_{2}(x), r_{3}(x), s_{2}(x), s_{3}(x) \in \mathbb{K}[x]$ provided that the denominator does not vanish. This example appears in [2, Example 3.8a]; see also Example 3.11 for details on these calculations.

The problem of describing the admissible degrees for the interpolation problem is tackled in [1] for $\delta$, and in [2] for $\kappa$. The main tool in the former paper is a divided-differences matrix, whereas the latter uses the Euclidean Algorithm to solve this problem. Later, in [3] the solutions of the classical (i.e., when $n_{i}=1$ for all $i=1, \ldots, l)$ rational interpolation problem are given as the kernel of a matrix encoding the data of the problem. In [13] this matrix is homogenized, and the numerical invariants of a minimal free resolution of its cokernel used to tackle the problem. 
In this paper, we connect the results obtained in [13 via resolutions of ideals and syzygies with the now standard and fast approach given by the Extended Euclidean Algorithm (EEA). As a consequence, we give explicit descriptions for both $\delta$ and $\kappa$ minimal degrees in terms of the degrees of elements appearing in the EEA. Of course, if one is interested in a fast method to solve any instance of the interpolation problem, the EEA is the most efficient tool available, and any improvement in dealing with this problem will also get translated into a faster algorithm to solve the EEA. In this sense, the two situations (solving the rational interpolation problem and computing the EEA of two polynomials) are equivalent from an algorithmic and complexity point of view. But this is not our focus. We are interested in exploring connections with syzygies and free resolutions because in the last decades these tools have been used to deal with other kinds of geometric problems like implicitization of rational parametrizations, or the description of some invariants associated to them. Hence, any dictionary between these methods brings the potential of shedding some light on situations different from the rational interpolation problem per se. As an example of this phenomenon, in Section 4 we obtain a simple description of the value of $\mu$ for a $\mu$-basis of a polynomial plane parametrization. This also shows that one can compute both $\mu$ and the $\mu$-basis considerably faster. It would be interesting to explore whether this result can be generalized for any rational parametrization, as it would considerably reduce the complexity of computing $\mu$ and $\mu$-bases. For the state of the art in this area, see [9] and the references therein.

We also treat with our methods the Hermite rational interpolation problem: for a given $d \in \mathbb{N}, 0 \leq d<n$, decide if there exist, and if so compute, polynomials $a(x)$, $b(x)$ of degrees bounded by $d$ and $n-d-1$ respectively, such that $\frac{a(x)}{b(x)}$ interpolates the data (see [6] for more on this problem). This problem is investigated at the end of Section 5

This paper is organized as follows. In Section 2 we review some basics on the Hermite interpolating polynomial and the Euclidean Algorithm. In Section 3 we introduce the language of syzygies, which helps us obtain a minimal basis associated to the rational interpolation problem. We show in Theorem 3.7 -which is a generalization of [3. Theorem 2.11] to the case of interpolation with multiplicitiesthat these minimal bases allow us to make the $\delta$-minimal degree explicit. Then we turn to make even more explicit this invariant by means of the EEA. This result is the content of Theorem 3.10, where we extract a minimal basis from some critical value in the sequence of degrees in the EEA.

In Section 4 we apply these results to make explicit the value of $\mu$ for a $\mu$-basis of a plane polynomial parametrization. In Theorem 4.1 this $\mu$ is expressed as a critical value in the sequence of degrees in a suitable EEA. We conclude this paper by applying our tools to study the $\kappa$-degree in Section 5 where we recover the results of Antoulas in [2] with our methods. 


\section{Hermite interpolating polynomial and Euclidean algorithm}

Throughout this paper $\mathbb{K}$ is an infinite field of characteristic either zero or larger than $\max \left\{n_{1}, \ldots, n_{l}\right\}$. This assumption ensures the existence of the Hermite interpolating polynomial associated to (1.1), which is the unique polynomial interpolating this data having degree smaller than $n$. We denote this interpolating polynomial by $g(x)$.

It is straightforward to check that any rational interpolating function $y(x)=\frac{a(x)}{b(x)}$ satisfies

$$
(a(x)-b(x) g(x))^{(j)}\left(x_{i}\right)=0, \quad i=1, \ldots, l, j=0, \ldots, n_{i}-1,
$$

and so $\left(x-x_{i}\right)^{n_{i}}$ divides $a(x)-b(x) g(x)$ for $i=1, \ldots, l$. Thus, if we set $f(x):=$ $\left(x-x_{1}\right)^{n_{1}} \cdots\left(x-x_{l}\right)^{n_{l}}$, there exists $c(x) \in \mathbb{K}[x]$ such that

$$
a(x)=b(x) g(x)+c(x) f(x) .
$$

In fact, 2.1 and 2.2 are equivalent, and we refer to any of these conditions as the weak interpolation conditions. Then $y(x)=\frac{a(x)}{b(x)}$ is an interpolating function if and only if the pair $(a(x), b(x))$ satisfies the weak interpolation conditions and $b\left(x_{i}\right) \neq 0$, for $i=1, \ldots, l$.

The next result follows easily.

Proposition 2.1. If the pair $(a(x), b(x))$ defines an interpolating function, then, for any polynomial $c(x)$ without roots in the set $\left\{x_{1}, \ldots, x_{l}\right\}$, the pair $(a(x) c(x)$, $b(x) c(x))$ also defines an interpolating function. Conversely, if $(a(x) c(x), b(x) c(x))$ defines an interpolating function, then $(a(x), b(x))$ defines an interpolating function.

Another way of stating Proposition 2.1 is that there exists an interpolating function of the data in a given class of $\mathbb{K}(x)$ if and only if the irreducible fraction of the class is an interpolating fraction. In particular, if $y(x)$ is an interpolating function, then there exist coprime polynomials $a(x), b(x)$ such that $y(x)=\frac{a(x)}{b(x)}$ in $\mathbb{K}(x)$.

Consider now two polynomials $r_{0}(x), r_{1}(x) \in \mathbb{K}[x]$ with $\operatorname{deg} r_{0}(x) \geq \operatorname{deg} r_{1}(x) \geq$ 0. By the Euclidean Algorithm, there exist a positive integer $N$ and unique nonzero polynomials $r_{2}(x), \ldots, r_{N}(x), q_{1}(x), \ldots, q_{N}(x)$ with $\operatorname{deg} r_{i}(x)>\operatorname{deg} r_{i+1}(x), i=$ $1, \ldots, N$, such that

$$
r_{i}(x)=q_{i+1}(x) r_{i+1}(x)+r_{i+2}(x), \quad i=0, \ldots, N-1,
$$

where $N$ is such that $r_{N}(x) \neq 0$ and $r_{N+1}(x)=0$.

Note that $\operatorname{deg} q_{i}(x)>0$ for all $i>1$ and that $\operatorname{deg} q_{i}(x)>0$ for all $i \geq 1$ if $\operatorname{deg} r_{0}(x)>\operatorname{deg} r_{1}(x)$. 
Using the quotients $q_{i}(x)$, we define recursively two sequences of polynomials $s_{i}(x), t_{i}(x)$, for $i=0, \ldots, N+1$, as follows:

$$
\begin{aligned}
\left(\begin{array}{l}
s_{0}(x) \\
t_{0}(x)
\end{array}\right) & =\left(\begin{array}{l}
0 \\
1
\end{array}\right), \quad\left(\begin{array}{l}
s_{1}(x) \\
t_{1}(x)
\end{array}\right)=\left(\begin{array}{l}
1 \\
0
\end{array}\right), \\
\left(\begin{array}{l}
s_{i}(x) \\
t_{i}(x)
\end{array}\right) & =\left(\begin{array}{l}
s_{i-2}(x) \\
t_{i-2}(x)
\end{array}\right)-q_{i-1}(x)\left(\begin{array}{l}
s_{i-1}(x) \\
t_{i-1}(x)
\end{array}\right),
\end{aligned}
$$

with $\operatorname{deg} s_{i}(x)>\operatorname{deg} s_{i-1}(x)$ and $\operatorname{deg} t_{i}(x)>\operatorname{deg} t_{i-1}(x)$ for $i>2$. We deduce from (2.3) and 2.4 that

$$
r_{i}(x)=r_{1}(x) s_{i}(x)+r_{0}(x) t_{i}(x), \quad i=0, \ldots, N+1 .
$$

Remark 2.2. An easy inductive argument on 2.3) and 2.4 yields

(a) $\operatorname{deg} r_{i}(x)=\operatorname{deg} r_{0}(x)-\left(\operatorname{deg} q_{1}(x)+\cdots+\operatorname{deg} q_{i}(x)\right)$, for $i=1, \ldots, N$.

(b) $\operatorname{deg} s_{i}(x)=\operatorname{deg} q_{1}(x)+\cdots+\operatorname{deg} q_{i-1}(x)$, for $i=2, \ldots, N$.

Lemma 2.3 ([2, Lemma 2.5]). Assume that $\operatorname{deg} r_{0}(x)>\operatorname{deg} r_{1}(x)$. For every $a(x), b(x), c(x) \in \mathbb{K}[x]$ satisfying $a(x)=r_{1}(x) b(x)+r_{0}(x) c(x)$, there exist unique polynomials $m_{0}(x), m_{1}(x), \ldots, m_{N+1}(x)$ such that

$$
\left(\begin{array}{l}
a(x) \\
b(x) \\
c(x)
\end{array}\right)=\sum_{i=0}^{N+1} m_{i}(x)\left(\begin{array}{l}
r_{i}(x) \\
s_{i}(x) \\
t_{i}(x)
\end{array}\right)
$$

with $\operatorname{deg} m_{i}(x)<\operatorname{deg} q_{i}(x), i=1, \ldots, N$.

Note that there are no bounds on the degrees of either $m_{0}(x)$ or $m_{N+1}(x)$ in Lemma 2.3

\section{SyzYGIES AND THE $\delta$-DEGREE}

For an interpolating function $y(x)=\frac{a(x)}{b(x)}$ of 1.1 there exists, by using 2.2, $c(x) \in \mathbb{K}[x]$ such that $a(x)=g(x) b(x)+c(x) f(x)$. So, $(a(x), b(x), c(x)) \in \mathbb{K}[x]^{3}$ is in the kernel of the following morphism of $\mathbb{K}[x]$-modules:

$$
\varphi: \mathbb{K}[x]^{3} \stackrel{(1-g(x)-f(x))}{\longrightarrow} \mathbb{K}[x] .
$$

The following result is a straightforward consequence of applying the Euclidean Algorithm to a solution of an equation of the form

$$
a(x)=g(x) b(x)+c(x) f(x) .
$$

Proposition 3.1. The kernel of $\varphi$ is a free module of rank 2 , and has $\{(f(x), 0,1)$, $(g(x), 1,0)\}$ as a basis.

Note that, in particular, we have

$$
(a(x), b(x))=\frac{a(x)-b(x) g(x)}{f(x)}(f(x), 0)+b(x)(g(x), 1),
$$

and that from here we deduce easily that $(f(x), 0)$ and $(g(x), 1)$ is also a basis of the $\mathbb{K}[x]$-submodule of $\mathbb{K}[x]^{2}$ defined by

$$
Y:=\left\{(a(x), b(x)) \in \mathbb{K}[x]^{2} \text { such that } a(x)-b(x) g(x) \in f(x) \mathbb{K}[x]\right\} .
$$


From its definition, it is clear that $Y$ is the set of the pairs of polynomials satisfying the weak interpolating conditions 2.1) or 2.2.

Homogenizing the above situation with a second variable $z$, we obtain a homogeneous morphism $\phi: \mathbb{K}[x, z](-n)^{3} \longrightarrow \mathbb{K}[x, z]$ given by the matrix

$$
\left(\begin{array}{lll}
z^{n} & -g(x, z) & -f(x, z)
\end{array}\right)
$$

and, by the Hilbert Syzygy Theorem, a minimal free resolution of $\mathbb{K}[x, z] / \operatorname{Coker}(\phi)$

$$
0 \longrightarrow \mathbb{K}[x, z]\left(-n-\mu_{1}\right) \oplus \mathbb{K}[x, z]\left(-n-\mu_{2}\right) \longrightarrow \mathbb{K}[x, z](-n)^{3} \stackrel{\phi}{\longrightarrow} \mathbb{K}[x, z],
$$

with $\mu_{1}+\mu_{2}=n$ and $\mu_{1} \leq \mu_{2}$. Our goal is to make explicit $\mu_{1}$ and $\mu_{2}$ by means of syzygies and the Euclidean Algorithm.

Let $\left\{\left(a_{1}(x, z), b_{1}(x, z), c_{1}(x, z)\right),\left(a_{2}(x, z), b_{2}(x, z), c_{2}(x, z)\right)\right\}$ be a basis of the kernel of $\phi$ such that $\operatorname{deg}\left(a_{i}(x, z), b_{i}(x, z), c_{i}(x, z)\right)=\mu_{i}, i=1,2$. Any relation or syzygy among $z^{n},-g(x, z)$, and $-f(x, z)$ can be written uniquely as a polynomial combination of these two triplets. In particular, for any homogeneous syzygy $(a(x, z), b(x, z), c(x, z))$ of degree $\delta$ there exist unique homogeneous polynomials $p(x, z), q(x, z)$, of degrees $\delta-\mu_{1}$ and $\delta-\mu_{2}$ respectively, or zero polynomials, such that

$$
\begin{aligned}
(a(x, z), b(x, z), c(x, z))= & p(x, z) \cdot\left(a_{1}(x, z), b_{1}(x, z), c_{1}(x, z)\right) \\
& +q(x, z) \cdot\left(a_{2}(x, z), b_{2}(x, z), c_{2}(x, z)\right) .
\end{aligned}
$$

Now set $a_{i}(x)=a_{i}(x, 1), b_{i}(x)=b_{i}(x, 1), c_{i}(x)=c_{i}(x, 1)$, for $i=1,2$.

Lemma 3.2. For any $(a(x), b(x), c(x))$ in the kernel of $\varphi$,

$$
\delta:=\max \{\operatorname{deg} a(x), \operatorname{deg} b(x), \operatorname{deg} c(x)\}=\max \{\operatorname{deg} a(x), \operatorname{deg} b(x)\},
$$

and there exist unique polynomials $p(x), q(x)$ such that

$$
(a(x), b(x))=p(x)\left(a_{1}(x), b_{1}(x)\right)+q(x)\left(a_{2}(x), b_{2}(x)\right) .
$$

Moreover, $\operatorname{deg} p(x) \leq \delta-\mu_{1}$ and $\operatorname{deg} q(x) \leq \delta-\mu_{2}$.

Proof. Let $k=\operatorname{deg} g(x)$. Note that $k \leq n-1<n$. Suppose that $\operatorname{deg} c(x)>$ $\max \{\operatorname{deg} a(x)$,

$\operatorname{deg} b(x)\}$. Then we would have that

$\operatorname{deg}(c(x) f(x))=\operatorname{deg} c(x)+n>\max \{\operatorname{deg} a(x), \operatorname{deg} b(x)+k\} \geq \operatorname{deg}(a(x)-b(x) g(x))$,

a contradiction with $(2.2)$, so the first part of the claim holds. For the second part, we homogenize $a(x), b(x), c(x)$ to degree $\delta$ and get $(3.2)$ with $p(x, z), q(x, z) \in$ $\mathbb{K}[x, z]$ of respective degrees $\delta-\mu_{1}$ and $\delta-\mu_{2}$. To get (3.3) we set $z=1$, and the claim follows straightforwardly.

Definition 3.3. We say that a basis $\left\{\left(a_{1}(x), b_{1}(x)\right),\left(a_{2}(x), b_{2}(x)\right)\right\}$ of $Y$ is minimal (or of minimal degree) if $\max \left\{\operatorname{deg} a_{i}(x), \operatorname{deg} b_{i}(x)\right\}=\mu_{i}, i=1,2$.

Note that, in general, $\{(f(x), 0),(g(x), 1)\}$ is not a minimal basis. 
Remark 3.4. Let $\left\{\left(a_{1}(x), b_{1}(x)\right),\left(a_{2}(x), b_{2}(x)\right)\right\}$ be a minimal basis of $Y$. From Definition 3.3 we deduce that for any $(a(x), b(x)) \in Y$, there exist unique $p(x)$, $q(x) \in \mathbb{K}[x]$ such that

$$
\begin{aligned}
(a(x), b(x)) & =p(x)\left(a_{1}(x), b_{1}(x)\right)+q(x)\left(a_{2}(x), b_{2}(x)\right) \\
& =\left(p(x) a_{1}(x)+q(x) a_{2}(x), p(x) b_{1}(x)+q(x) b_{2}(x)\right) .
\end{aligned}
$$

Let $c(x) \in \mathbb{K}[x]$ be the polynomial such that 2.2 holds, and set $\delta:=\max \{\operatorname{deg} a(x)$, $\operatorname{deg} b(x)\}$. Homogenizing this situation, thanks to Lemma 3.2 we have

$\delta=\max \left\{\operatorname{deg}\left(p(x, z) a_{1}(x, z)+q(x, z) a_{2}(x, z)\right), \operatorname{deg}\left(p(x, z) b_{1}(x, z)+q(x, z) b_{2}(x, z)\right)\right\}$,

and from here we deduce that $\operatorname{deg} p(x) \leq \delta-\mu_{1}$ and $\operatorname{deg} q(x) \leq \delta-\mu_{2}$. In particular, $\delta\left(\frac{a(x)}{b(x)}\right) \geq \mu_{1}$, and if $q(x) \neq 0$, then $\delta\left(\frac{a(x)}{b(x)}\right) \geq \mu_{2}$.

Remark 3.5. Any basis of $Y$ with $\delta$-degrees $\delta_{1}, \delta_{2}$ with $\delta_{1}+\delta_{2}=n$ is a minimal basis of $Y$. In this case, $\left\{\delta_{1}, \delta_{2}\right\}=\left\{\mu_{1}, \mu_{2}\right\}$.

Remark 3.6. Let $\left\{\left(a_{1}(x), b_{1}(x)\right),\left(a_{2}(x), b_{2}(x)\right)\right\}$ be a minimal basis of $Y$. From Proposition 2.1 we deduce that the unique possible irreducible common factors of $a_{i}(x)$ and $b_{i}(x)$ are of the form $x-x_{j}$ for some $j=1, \ldots, l ; i=1,2$. Otherwise, by removing such a factor, we would obtain a pair satisfying the weak interpolating conditions with degree strictly smaller than $\mu_{1}$ or than $\mu_{2}$.

Also, since there exist interpolating functions for any data (for instance, the interpolating polynomial $g(x)), b_{1}(x)$ and $b_{2}(x)$ cannot both vanish at $x_{i}$ for any $i=1, \ldots, l$.

The following is the main result of this section, which generalizes [3, Theorem $2.11]$ to the case of interpolation with multiplicities.

Theorem 3.7. Let $\left\{\left(a_{1}(x), b_{1}(x)\right),\left(a_{2}(x), b_{2}(x)\right)\right\}$ be a minimal basis of $Y$. The rational function $y(x)$ is an interpolating function if and only if there exist polynomials $p(x)$ and $q(x)$ such that

$$
y(x)=\frac{p(x) a_{1}(x)+q(x) a_{2}(x)}{p(x) b_{1}(x)+q(x) b_{2}(x)}
$$

with $p\left(x_{i}\right) b_{1}\left(x_{i}\right)+q\left(x_{i}\right) b_{2}\left(x_{i}\right) \neq 0$ for $i=1, \ldots, l$.

If $a_{1}(x)$ and $b_{1}(x)$ are coprime and $\mu_{1}<\mu_{2}$, then there is a unique interpolating function $y_{\min }(x)$ of minimal degree $\mu_{1}$ given by

$$
y_{\min }(x)=\frac{a_{1}(x)}{b_{1}(x)} .
$$

Otherwise (i.e., if either $a_{1}(x)$ and $b_{1}(x)$ are not coprime, or $\mu_{1}=\mu_{2}$ ), there is a family of interpolating functions of minimal degree $\mu_{2}$ which can be parametrized as

$$
y_{\min }(x)=\frac{a_{2}(x)+p(x) a_{1}(x)}{b_{2}(x)+p(x) b_{1}(x)},
$$

where $\operatorname{deg} p(x)=\mu_{2}-\mu_{1}$, and $b_{2}\left(x_{i}\right)+p\left(x_{i}\right) b_{1}\left(x_{i}\right) \neq 0, i=1, \ldots, l$. 
Proof. The rational function $y(x)=\frac{a(x)}{b(x)}$ interpolates 1.1 if and only if $b\left(x_{i}\right) \neq$ 0 for all $i=1, \ldots, l$, and it satisfies the weak interpolation conditions of $(2.2)$. Equivalently we must have $(a(x), b(x)) \in Y$, and $b\left(x_{i}\right) \neq 0$ for $i=1, \ldots, l$. From here we deduce the first part of the claim thanks to Remark 3.4

Assume now that $\mu_{1}<\mu_{2}$ and $a_{1}(x)$ and $b_{1}(x)$ are coprime. If $b_{1}\left(x_{i}\right)=0$ for some $i$, then also $a_{1}\left(x_{i}\right)=0$, since $a_{1}(x)-b_{1}(x) g(x) \in f(x) \mathbb{K}[x]$, which contradicts the assumption that $a_{1}(x), b_{1}(x)$ are coprime. The rational function $y(x)=\frac{a_{1}(x)}{b_{1}(x)}$ interpolates the data in this case. Moreover, by Remark 3.4 $y(x)$ is the unique rational function with minimum degree that interpolates the data.

If $\mu_{1}<\mu_{2}$ and $a_{1}(x)$ and $b_{1}(x)$ are not coprime then, by using Remark 3.6. $b_{1}\left(x_{i}\right)=0$ for some $i=1, \ldots, l$ and $\frac{a_{1}(x)}{b_{1}(x)}$ does not interpolate the data. In this case, any pair $\left(p(x) a_{1}(x)+q(x) a_{2}(x), p(x) b_{1}(x)+q(x) b_{2}(x)\right)$ defining an interpolating function must have $q(x) \neq 0$ and degree at least $\mu_{2}$. So, the interpolating functions of minimal degree are parametrized by $\frac{a_{2}(x)+p(x) a_{1}(x)}{b_{2}(x)+p(x) b_{1}(x)}$, where $p(x)$ is a polynomial of degree $\mu_{2}-\mu_{1}$ provided that $b_{2}\left(x_{i}\right)+p\left(x_{i}\right) b_{1}\left(x_{i}\right) \neq 0, i=1, \ldots, l$. It is straightforward to verify that there is at least one such polynomial. Indeed, by choosing $\lambda \in \mathbb{K} \backslash\left\{-\frac{b_{2}\left(x_{i}\right)}{b_{1}\left(x_{i}\right)}, i=1, \ldots, l, b_{1}\left(x_{i}\right) \neq 0\right\}$, which can be done thanks to Remark 3.6, and by using the fact that $\mathbb{K}$ has infinitely many elements, the polynomial $b_{2}(x)+\lambda b_{1}(x)$ satisfies the claim. Note that for these values of $\lambda$ it cannot happen that $\delta\left(\frac{a_{2}(x)+\lambda a_{1}(x)}{b_{2}(x)+\lambda b_{1}(x)}\right)<\mu_{2}$, as this would imply that the pair $\left(a_{2}(x)+\lambda a_{1}(x), b_{2}(x)+\lambda b_{1}(x)\right)$ is a polynomial multiple of $\left(a_{1}(x), b_{1}(x)\right)$, which is impossible since $b_{1}(x)$ vanishes at some of the $x_{i}$ 's and $\lambda$ has been chosen in such a way that $b_{2}(x)+\lambda b_{1}(x)$ does not vanish at any of these points.

If $\mu_{1}=\mu_{2}$, the family of interpolating rational functions of minimal degree can be written as $\frac{a_{2}(x)+\lambda a_{1}(x)}{b_{2}(x)+\lambda b_{1}(x)}$, with $\lambda \in \mathbb{K} \backslash\left\{-\frac{b_{2}\left(x_{i}\right)}{b_{1}\left(x_{i}\right)}, i=1, \ldots, l, b_{1}\left(x_{i}\right) \neq 0\right\}$, as before. This concludes the proof of the theorem.

Corollary 3.8. The minimal $\delta$-admissible degree is either $\mu_{1}$ or $\mu_{2}$. The set of $\delta$-admissible degrees is either $\left\{\mu_{1}\right\} \cup\left\{\delta \geq \mu_{2}\right\}$ or $\left\{\delta \geq \mu_{2}\right\}$.

Proof. By Theorem 3.7 the minimal admissible $\delta$-degree of $y(x)$ is either $\mu_{1}$ or $\mu_{2}$. Moreover, any $y(x)=\frac{a(x)}{b(x)}$ interpolating function with $\delta$-degree $\geq \mu_{2}$ can be written as

$$
\frac{a(x)}{b(x)}=\frac{p(x) a_{1}(x)+q(x) a_{2}(x)}{p(x) b_{1}(x)+q(x) b_{2}(x)}
$$

for some $p(x), q(x) \in \mathbb{K}[x], q(x) \neq 0$.

We have already seen in Theorem 3.7 that $\mu_{1}$ may be admissible, and also that $\mu_{2}$ is admissible if $\mu_{1}$ is not. Moreover, as in the proof of Theorem 3.7 it is easy to check that there cannot be any rational function as in $(3.4)$ of $\delta$-degree strictly larger than $\mu_{1}$ and smaller than $\mu_{2}$, since this would imply that $\left(p(x) a_{1}(x)+\right.$ $\left.q(x) a_{2}(x), p(x) b_{1}(x)+q(x) b_{2}(x)\right)$ is a polynomial multiple of $\left(a_{1}(x), b_{1}(x)\right)$, and hence it would have $\delta$-degree equal to $\mu_{1}$, a contradiction. 
To see that any $\delta \geq \mu_{2}$ is admissible, pick $\frac{\tilde{a}(x)}{\tilde{b}(x)}$ a minimal solution of $\delta$-degree either $\mu_{1}$ or $\mu_{2}$. Note that this implies $\operatorname{gcd}(\tilde{a}(x), \tilde{b}(x))=1$. Now set

$$
(a(x), b(x)):=\left(\tilde{a}(x)+\lambda x^{\delta-\mu_{2}} a_{2}(x), \tilde{b}(x)+\lambda x^{\delta-\mu_{2}} b_{2}(x)\right),
$$

for a suitable $\lambda \in \mathbb{K} \backslash\{0\}$ such that

$$
\operatorname{gcd}\left(\tilde{a}(x)+\lambda x^{\delta-\mu_{2}} a_{2}(x), \tilde{b}(x)+\lambda x^{\delta-\mu_{2}} b_{2}(x)\right)=1 .
$$

This can be done because (3.5) is equivalent to the fact that the resultant of the polynomials $\tilde{a}(x)+\lambda x^{\delta-\mu_{2}} a_{2}(x)$ and $\tilde{b}(x)+\lambda x^{\delta-\mu_{2}} b_{2}(x)$ does not vanish identically. This resultant is a polynomial in $\lambda$ whose constant coefficient is equal to $\operatorname{Res}(\tilde{a}(x), \tilde{b}(x)) \neq 0$ as these polynomials do not share any common factor. So,

$$
\operatorname{Res}\left(\tilde{a}(x)+\lambda x^{\delta-\mu_{2}} a_{2}(x), \tilde{b}(x)+\lambda x^{\delta-\mu_{2}} b_{2}(x)\right) \in \mathbb{K}[\lambda]
$$

is not the zero polynomial, and by choosing a $\lambda \in \mathbb{K}$ which is not a zero of this polynomial (this can be done because $\mathbb{K}$ is infinite), the claim follows for $\delta \geq \mu_{2}$.

In what follows, we are going to make explicit the $\mu_{i}$ 's by means of the Extended Euclidean Algorithm.

3.1. Minimal basis and the Euclidean algorithm. Following the notation in Section 2 for $r_{0}(x)=f(x)$ and $r_{1}(x)=g(x)$, we can proceed as in 3 . In that paper, the authors deal with the rational interpolation problem without multiplicities, to obtain a minimal basis from the Euclidean algorithm. We show here that the same approach works for the general case.

The vector relations

$$
\left(\begin{array}{c}
r_{i+2}(x) \\
s_{i+2}(x) \\
t_{i+2}(x)
\end{array}\right)=\left(\begin{array}{c}
r_{i}(x) \\
s_{i}(x) \\
t_{i}(x)
\end{array}\right)-q_{i+1}(x)\left(\begin{array}{c}
r_{i+1}(x) \\
s_{i+1}(x) \\
t_{i+i}(x)
\end{array}\right)
$$

with the initial conditions

$$
\left(\begin{array}{c}
r_{0}(x) \\
s_{0}(x) \\
t_{0}(x)
\end{array}\right)=\left(\begin{array}{c}
f(x) \\
0 \\
1
\end{array}\right), \quad\left(\begin{array}{c}
r_{1}(x) \\
s_{1}(x) \\
t_{1}(x)
\end{array}\right)=\left(\begin{array}{c}
g(x) \\
1 \\
0
\end{array}\right)
$$

produce a sequence of elements in the kernel of $\left(\begin{array}{lll}1 & -g(x) & -f(x)\end{array}\right)$.

Proposition 3.9. For $0 \leq i \leq N-1$, the set

$$
\left\{\left(r_{i}(x), s_{i}(x), t_{i}(x)\right),\left(r_{i+1}(x), s_{i+1}(x), t_{i+1}(x)\right)\right\} \subset \mathbb{K}[x]^{3}
$$

is a basis of the kernel of $\varphi$. Also, $\left\{\left(r_{i}(x), s_{i}(x)\right),\left(r_{i+1}(x), s_{i+1}(x)\right)\right\}$ is a basis of $Y$.

Proof. We will use induction on $i$. The case $i=0$ is given by (3.7). For the general case, we just have to show that one can replace the triplet $\left(r_{i}(x), s_{i}(x), t_{i}(x)\right)$ in the basis $\left\{\left(r_{i}(x), s_{i}(x), t_{i}(x)\right),\left(r_{i+1}(x), s_{i+1}(x), t_{i+1}(x)\right)\right\}$ with $\left(r_{i+2}(x), s_{i+2}(x), t_{i+2}(x)\right)$ and still generate the same kernel. But this follows straightforwardly thanks to (3.6), which proves the first part of the claim. The rest holds by projecting onto the first two coordinates the previous result, and using Remark 3.4 
From (3.6) we deduce that, for $i=1, \ldots, N$,

$$
\begin{aligned}
& \left(\begin{array}{cc}
\operatorname{deg} r_{i}(x) & \operatorname{deg} r_{i+1}(x) \\
\operatorname{deg} s_{i}(x) & \operatorname{deg} s_{i+1}(x)
\end{array}\right) \\
& =\left(\begin{array}{cc}
n-\left(\operatorname{deg} q_{1}(x)+\cdots+\operatorname{deg} q_{i}(x)\right) & n-\left(\operatorname{deg} q_{1}(x)+\cdots+\operatorname{deg} q_{i+1}(x)\right) \\
\operatorname{deg} q_{1}(x)+\cdots+\operatorname{deg} q_{i-1}(x) & \operatorname{deg} q_{1}(x)+\cdots+\operatorname{deg} q_{i}(x)
\end{array}\right) .
\end{aligned}
$$

Theorem 3.10. Let $i \in\{1, \ldots, N\}$ be such that

$$
n-\sum_{j=1}^{i} \operatorname{deg} q_{j}(x) \geq \sum_{j=1}^{i-1} \operatorname{deg} q_{j}(x) \quad \text { and } \quad \sum_{j=1}^{i} \operatorname{deg} q_{j}(x) \geq n-\sum_{j=1}^{i+1} \operatorname{deg} q_{j}(x)
$$

or, equivalently,

$$
2\left(\sum_{j=1}^{i-1} \operatorname{deg} q_{j}(x)\right)+\operatorname{deg} q_{i}(x) \leq n \leq 2\left(\sum_{j=1}^{i} \operatorname{deg} q_{j}(x)\right)+\operatorname{deg} q_{i+1}(x) .
$$

Then $\left\{\left(r_{i}(x), s_{i}(x)\right),\left(r_{i+1}(x), s_{i+1}(x)\right)\right\}$ is a minimal basis of $Y$.

Proof. From Proposition 3.9 we know that $\left\{\left(r_{i}(x), s_{i}(x)\right),\left(r_{i+1}(x), s_{i+1}(x)\right)\right\}$ is a basis of $Y$. For $j=0,1$, let $\delta_{j}=\max \left\{\operatorname{deg} r_{i+1-j}(x), \operatorname{deg} s_{i+1-j}(x)\right\}$.

From 3.8 and $(3.9)$, we deduce that

$$
\delta_{1}+\delta_{2}=\left(n-\sum_{j=1}^{i} \operatorname{deg} q_{j}(x)\right)+\left(\sum_{j=1}^{i} \operatorname{deg} q_{j}(x)\right)=n .
$$

The claim now follows thanks to Remark 3.5 .

Example 3.11. Consider

$$
\begin{array}{ll}
x_{1}=0, & y_{1,0}=-2 \\
x_{2}=2, & y_{2,0}=6 \\
x_{3}=-1, & y_{3,0}=-3 \\
x_{3}=-1, & y_{3,1}=3 .
\end{array}
$$

In this case, we have $N=3$ and the sequences of polynomials produced by the Euclidean Algorithm are

\begin{tabular}{c|c|c|c|c}
$i$ & 0 & 1 & 2 & 3 \\
\hline$r_{i}(x)$ & $x^{4}-3 x^{2}-2 x$ & $x^{3}-2$ & $-3 x^{2}$ & -2 \\
\hline$s_{i}(x)$ & 0 & 1 & $-x$ & $\frac{-x^{2}}{3}+1$ \\
\hline
\end{tabular}

with $q_{1}(x)=x, q_{2}(x)=-\frac{x}{3}, q_{3}(x)=\frac{3 x^{2}}{2}$.

So, $\mu_{1}=\mu_{2}=2,\left\{\left(r_{2}(x), s_{2}(x)\right),\left(r_{3}(x), s_{3}(x)\right)\right\}$ is a minimal basis, the $\delta$-minimal degree is 2 , and the fractions $\frac{-2-3 \lambda x^{2}}{\frac{-x^{2}}{3}+1-\lambda x}$ define the minimal interpolating functions for all $\lambda \in \mathbb{K}, \lambda \neq-\frac{1}{6},-\frac{2}{3}$. 
The interpolating functions $y(x)=\frac{a(x)}{b(x)}$ with $\delta(y(x))=\delta>2$ can be parametrized as

$$
y(x)=\frac{\left(\lambda_{0}+\cdots+\lambda_{\delta-2} x^{\delta-2}\right) r_{2}(x)+\left(\lambda_{0}^{\prime}+\cdots+\lambda_{\delta-3}^{\prime} x^{\delta-3}+x^{\delta-2}\right) r_{3}(x)}{\left(\lambda_{0}+\cdots+\lambda_{\delta-2} x^{\delta-2}\right) s_{2}(x)+\left(\lambda_{0}^{\prime}+\cdots+\lambda_{\delta-3}^{\prime} x^{\delta-3}+x^{\delta-2}\right) s_{3}(x)},
$$

provided that $\left(\lambda_{0}+\cdots+\lambda_{\delta-2} x_{i}^{\delta-2}\right) s_{2}\left(x_{i}\right)+\left(\lambda_{0}^{\prime}+\cdots+\lambda_{\delta-3}^{\prime} x_{i}^{\delta-3}+x_{i}^{\delta-2}\right) s_{3}\left(x_{i}\right) \neq 0$, for $i=1,2,3$.

The index $i$ in Theorem 3.10 is not unique, although there are at most two possible choices for this index, as the following cautionary example shows.

Example 3.12. Take the interpolating data $(1,1),(-1,1),(2,-14),(-2,-14)$, $(3,1),(-3,1)$. In this case, $N=3$, and the Euclidean Algorithm gives

\begin{tabular}{c|c|c|c|c}
$i$ & 0 & 1 & 2 & 3 \\
\hline$r_{i}(x)$ & $x^{6}-14 x^{4}+49 x^{2}-36$ & $x^{4}-10 x^{2}+10$ & $-x^{2}+4$ & -4 \\
\hline$s_{i}(x)$ & 0 & 1 & $-x^{2}+4$ & $-x^{4}+2 x^{2}-3$ \\
\hline
\end{tabular}

with $q_{1}(x)=x^{2}-4, q_{2}(x)=-x^{2}+6, q_{3}(x)=\frac{x^{2}-4}{14}$. So, $\mu_{1}=2, \mu_{2}=4$, and $\left\{\left(r_{1}(x), s_{1}(x)\right),\left(r_{2}(x), s_{2}(x)\right)\right\}$ is a minimal basis. The $\delta$-minimal degree is 4 , and

$$
\frac{x^{4}-10 x^{2}+10+\left(\lambda_{0}+\lambda_{1} x+\lambda_{2} x^{2}\right)\left(-x^{2}+4\right)}{1+\left(\lambda_{0}+\lambda_{1} x+\lambda_{2} x^{2}\right)\left(-x^{2}+4\right)}
$$

parametrizes the minimal rational interpolating functions, provided that the denominators do not vanish at the $x_{i}$ 's.

Note that another minimal basis is given by $\left\{\left(r_{2}(x), s_{2}(x)\right),\left(r_{3}(x), s_{3}(x)\right)\right\}$.

Example 3.13. In the generic case, that is, if all the quotients in the Euclidean Algorithm have degree one, we have $N=n, \operatorname{deg} r_{i}(x)=n-i$, and $\operatorname{deg} s_{i}(x)=i-1$ for $i=1, \ldots N$. From Theorem 3.10 we deduce straightforwardly that

(a) If $n=2 k$ then $\left\{\left(r_{k}(x), s_{k}(x)\right),\left(r_{k+1}(x), s_{k+1}(x)\right)\right\}$ is a minimal basis and the $\delta$-minimal degree is $k$.

(b) If $n=2 k+1$ then both $\left\{\left(r_{k}(x), s_{k}(x)\right),\left(r_{k+1}(x), s_{k+1}(x)\right)\right\}$ and $\left\{\left(r_{k+1}(x)\right.\right.$, $\left.\left.s_{k+1}(x)\right),\left(r_{k+2}(x), s_{k+2}(x)\right)\right\}$ are minimal bases, and the $\delta$-minimal degree is either $k$ or $k+1$.

Example 3.14. Consider the data $(-1,-3),(0,-2),(1,-1)$, and $(2,6)$. This is a generic case with an even number of pairs for $r_{0}(x)=f(x)=x^{4}-2 x^{3}-x^{2}+2 x$ and $r_{1}(x)=g(x)=x^{3}-2$.

\begin{tabular}{c|c|c|c|c|c}
$i$ & 0 & 1 & 2 & 3 & 4 \\
\hline$r_{i}(x)$ & $x^{4}-2 x^{3}-x^{2}+2 x$ & $x^{3}-2$ & $-x^{2}+4 x-4$ & $12 x-18$ & $\frac{-1}{4}$ \\
\hline$s_{i}(x)$ & 0 & 1 & $-x+2$ & $-x^{2}-2 x+9$ & $\frac{-8 x^{2}+4 x+3}{24}$ \\
\hline
\end{tabular}


The family of functions $\frac{12 x-18+\lambda\left(-x^{2}+4 x-4\right)}{-x^{2}-2 x+9+\lambda(-x+2)}$ with $\lambda \neq \frac{-10}{3}, \frac{-9}{2},-6$ interpolate with $\delta$-minimal degree equal to 2 .

\section{4. $\mu$-BASIS FOR POLYNOMIAL PARAMETRIZATIONS OF PLANE CURVES}

The approach used to find a minimal basis for the $\delta$-degree actually allows us to compute a $\mu$-basis for the parametrization of a polynomial planar curve, and also characterize the value of $\mu$ in terms of the critical degree $(3.9)$ arising in the Extended Euclidean Algorithm. Definitions and basic properties and applications of $\mu$-bases in the general setting of rational curves can be found in [7] (see also [9]). We briefly recall them here for polynomial parametrizations.

Let $r_{0}(x), r_{1}(x) \in \mathbb{K}[x]$, and consider the polynomial parametrization of an affine plane curve given by

$$
\begin{aligned}
\mathbb{K} & \rightarrow \mathbb{K}^{2} \\
t & \mapsto\left(r_{0}(t), r_{1}(t)\right) .
\end{aligned}
$$

Denote by $I$ the ideal in the polynomial ring $\mathbb{K}\left[x, T_{0}, T_{1}\right]$ defined by $T_{0}-r_{0}(x)$ and $T_{1}-r_{1}(x)$.

Let $I_{i, j}$ be the set of elements of $I$ with degree at most $i$ in $x$ and total degree at most $j$ in $T_{0}, T_{1}$. It is easy to see that a polynomial $a(x) T_{0}+b(x) T_{1}+c(x)$ with $a(x), b(x), c(x) \in \mathbb{K}[x]$ is in $I_{*, 1}$ if, and only if, $a(x) r_{0}(x)+b(x) r_{1}(x)+c(x)=0$, i.e., $(a(x), b(x), c(x))$ is a syzygy of $\left(r_{0}(x), r_{1}(x), 1\right)$. This implies that there exists an isomorphism of $\mathbb{K}[x]$-modules

$$
\begin{aligned}
I_{*, 1} & \longrightarrow \operatorname{Syz}\left(r_{0}(x), r_{1}(x), 1\right) \\
a(x) T_{0}+b(x) T_{1}+c(x) & \mapsto(a(x), b(x), c(x))
\end{aligned}
$$

Assume that $n=\operatorname{deg} r_{0}(x) \geq \operatorname{deg} r_{1}(x)$. Let $\mu$ be the smallest integer such that $I_{\mu, 1} \neq 0$. By homogenizing and applying the Hilbert Syzygy Theorem as in (3.1), there exist polynomials $p\left(x, T_{0}, T_{1}\right) \in I_{\mu, 1}$ and $q\left(x, T_{0}, T_{1}\right) \in I_{n-\mu, 1}$ such that every polynomial $a(x) T_{0}+b(x) T_{1}+c(x) \in I_{*, 1}$ can be written uniquely in the form

$$
a(x) T_{0}+b(x) T_{1}+c(x)=h_{1}(x) p\left(x, T_{0}, T_{1}\right)+h_{2}(x) q\left(x, T_{0}, T_{1}\right),
$$

with $h_{1}(x), h_{2}(x) \in \mathbb{K}[x]$. The polynomials $p\left(x, T_{0}, T_{1}\right), q\left(x, T_{0}, T_{1}\right)$ are called a $\mu$-basis of the parametrization 4.1.

Homogenizing 4.1, we have a map

$$
\begin{aligned}
\mathbb{P}^{1} & \rightarrow \mathbb{P}^{2} \\
\left(t_{0}: t_{1}\right) & \mapsto\left(r_{0}\left(t_{0}: t_{1}\right): r_{1}\left(t_{0}: t_{1}\right): t_{1}^{n}\right)
\end{aligned}
$$

whose image is a projective plane curve. We denote by

$$
\begin{aligned}
\mathcal{I}_{*, 1}=\left\{a(x, z) T_{0}+b(x, z) T_{1}\right. & +c(x, z) T_{2} \in \mathbb{K}\left[x, z, T_{0}, T_{1}, T_{2}\right] \\
& \left.: a(x, z) r_{0}(x, z)+b(x, z) r_{1}(x, z)+c(x, z) z^{n}=0\right\}
\end{aligned}
$$

the $\mathbb{K}[x, z]$-submodule of moving lines following the parametrization 4.2 . It is easy to verify that there is an isomorphism of graded $\mathbb{K}[x, z]$-modules

$$
\begin{aligned}
\mathcal{I}_{*, 1} & \longrightarrow \operatorname{Syz}\left(r_{0}(x, z), r_{1}(x, z), z^{n}\right) \\
a(x, z) T_{0}+b(x, z) T_{1}+c(x, z) T_{2} & \mapsto(a(x, z), b(x, z), c(x, z)) .
\end{aligned}
$$


Again by the Hilbert Syzygy Theorem we deduce that $\operatorname{Syz}\left(r_{0}(x, z), r_{1}(x, z), z^{n}\right)$ is a free module of rank 2 , with a homogeneous basis

$$
p=\left(p_{1}(x, z), p_{2}(x, z), p_{3}(x, z)\right), \quad q=\left(q_{1}(x, z), q_{2}(x, z), q_{3}(x, z)\right)
$$

of degrees $\mu$ and $n-\mu$ respectively, where we assume $\mu \leq n-\mu$. By dehomogenizing this situation, we deduce that

$$
\left\{p_{1}(x, 1) T_{0}+p_{2}(x, 1) T_{1}+p_{3}(x, 1), q_{1}(x, 1) T_{0}+q_{2}(x, 1) T_{1}+q_{3}(x, 1)\right\}
$$

is a $\mu$-basis of 4.1.

As in Section 2 let $r_{i}(x), s_{i}(x), t_{i}(x)$, for $i=0, \ldots, N+1$, be the sequences of polynomials from the Extended Euclidean Algorithm starting with $r_{0}(x)$ of degree $n$ and $r_{1}(x)$ of lower degree. From 3.1 we deduce that $\left(t_{i}(x), s_{i}(x),-r_{i}(x)\right)$ is a syzygy of $\left(r_{0}(x), r_{1}(x), 1\right)$ for all $i=0, \ldots, N$. The following result states the value of $\mu$ in terms of the degrees appearing in the Extended Euclidean Algorithm, and also extracts a $\mu$-basis of 4.1 from the sequence of remainders.

Theorem 4.1. There exists $i_{\mu} \in\{0,1, \ldots, N-1\}$ such that

$$
\max \left\{\operatorname{deg} r_{i_{\mu}}(x), \operatorname{deg} s_{i_{\mu}}(x)\right\}+\max \left\{\operatorname{deg} r_{i_{\mu}+1}(x), \operatorname{deg} s_{i_{\mu}+1}(x)\right\}=n .
$$

For this index, we have

$$
\mu=\min \left\{\max \left\{\operatorname{deg} r_{i_{\mu}}(x), \operatorname{deg} s_{i_{\mu}}(x)\right\}, \max \left\{\operatorname{deg} r_{i_{\mu}+1}(x), \operatorname{deg} s_{i_{\mu}+1}(x)\right\}\right\},
$$

and moreover $\left\{\left(t_{i_{\mu}}(x), s_{i_{\mu}}(x),-r_{i_{\mu}}(x)\right),\left(t_{i_{\mu}+1}(x), s_{i_{\mu}+1}(x),-r_{i_{\mu}+1}(x)\right)\right\}$ is a $\mu$-basis of 4.1 .

Proof. Choose as $i_{\mu}$ the one satisfying (3.9); then the first part of the claim follows directly from Theorem 3.10 For the rest, suppose without loss of generality that $\mu=\max \left\{\operatorname{deg} r_{i_{\mu}}(x), \operatorname{deg} s_{i_{\mu}}(x)\right\}$. From Lemma 3.2 we deduce that

$$
\mu=\max \left\{\operatorname{deg} t_{i_{\mu}}(x), \operatorname{deg} r_{i_{\mu}}(x), \operatorname{deg} s_{i_{\mu}}(x)\right\},
$$

and also that $n-\mu=\max \left\{\operatorname{deg} t_{i_{\mu}+1}(x), \operatorname{deg} r_{i_{\mu}+1}(x), \operatorname{deg} s_{i_{\mu}+1}(x)\right\}$. The fact that $\left\{\left(t_{i_{\mu}}(x), s_{i_{\mu}}(x),-r_{i_{\mu}}(x)\right),\left(t_{i_{\mu}+1}(x), s_{i_{\mu}+1}(x),-r_{i_{\mu}+1}(x)\right)\right\}$ is a $\mu$-basis of 4.1 follows then straightforwardly from Proposition 3.9

Example 4.2. For the planar affine curve parametrized by $\left(6 x^{2}-4 x^{4}, 4 x-4 x^{3}\right)$ we have

\begin{tabular}{c|c|c|c|c}
$i$ & 0 & 1 & 2 & 3 \\
\hline$r_{i}(x)$ & $-4 x^{4}+6 x^{2}$ & $-4 x^{3}+x$ & $2 x^{2}$ & $4 x$ \\
\hline$s_{i}(x)$ & 0 & 1 & $-x$ & $1-2 x^{2}$ \\
\hline$t_{i}(x)$ & 1 & 0 & 1 & $2 x$ \\
\hline
\end{tabular}

We deduce then that $\mu=2$ and that $\left\{T_{0}-x T_{1}-2 x^{2}, 2 x T_{0}+\left(1-2 x^{2}\right) T_{1}-4 x\right\}$ is a $\mu$-basis of the parametrization.

From the above, we have that for the projective planar curve parametrized by $\left(6 x^{2} z^{2}-4 x^{4}, 4 x z^{2}-4 x^{3}, z^{4}\right)$, the free $\mathbb{K}[x, z]$-module of moving lines following the 
parametrization is generated by $z^{2} T_{0}-x z T_{1}-2 x^{2} T_{2}$ and $2 x z T_{0}+\left(z^{2}-2 x^{2}\right) T_{1}-$ $4 x z T_{2}$.

Example 4.3. For the planar affine curve parametrized by $\left(x^{n}, x^{m}\right)$ with $m \leq n$, we have

\begin{tabular}{c|c|c|c}
$i$ & 0 & 1 & 2 \\
\hline$r_{i}(x)$ & $x^{n}$ & $x^{m}$ & 0 \\
\hline$s_{i}(x)$ & 0 & 1 & $-x^{n-m}$ \\
\hline$t_{i}(x)$ & 1 & 0 & 1 \\
\hline
\end{tabular}

From this table we deduce that $\mu=\min (m, n-m)$, and $\left\{T_{1}-x^{m}, T_{0}-x^{n-m} T_{1}\right\}$ is a $\mu$-basis of the parametrization.

For the corresponding projective plane curve parametrized by $\left(x^{n}, x^{m} z^{n-m}, z^{n}\right)$, the module of moving lines following the parametrization is generated by $z^{m} T_{1}-$ $x^{m} T_{2}$ and $z^{n-m} T_{0}-x^{n-m} T_{1}$.

\section{5 . THE $\kappa$-DEGREE}

Here we use again the notation of Section 2 and set $r_{0}(x)=f(x)$ and $r_{1}(x)=$ $g(x)$. The following result is obtained straightforwardly from the characterization given in 2.2 of the interpolating functions, and Lemma 2.3

Theorem 5.1. For every rational function $y(x)$ satisfying 1.2 , there exists a unique set of polynomials $m_{0}(x), \ldots, m_{N+1}(x)$ such that

$$
y(x)=\frac{\sum_{i=0}^{N+1} m_{i}(x) r_{i}(x)}{\sum_{i=0}^{N+1} m_{i}(x) s_{i}(x)}, \text { with } \operatorname{deg} m_{i}(x)<\operatorname{deg} q_{i}(x) \text { for } i=1, \ldots, N,
$$

and $\sum_{i=0}^{N+1} m_{i}\left(x_{j}\right) s_{i}\left(x_{j}\right) \neq 0$, for $j=1, \ldots l$.

Now we are ready to present the main result of this section, which recovers [2, Corollary 3.5].

Theorem 5.2. The set of admissible $\kappa$ 's is

$$
\left\{n-\operatorname{deg} q_{k}(x), s_{k}\left(x_{i}\right) \neq 0, \forall i=1, \ldots, l\right\} \cup\{j \geq n\} .
$$

The minimum admissible degree is

$$
\min _{k}\left\{n-\operatorname{deg} q_{k}(x), s_{k}\left(x_{i}\right) \neq 0, \forall i=1, \ldots, l\right\} .
$$

Proof. Given an interpolating rational function we write it, by using Theorem 5.1 . as

$$
y(x)=\frac{\sum_{i=0}^{N+1} m_{i}(x) r_{i}(x)}{\sum_{i=0}^{N+1} m_{i}(x) s_{i}(x)}
$$

with $\operatorname{deg} m_{i}(x)<\operatorname{deg} q_{i}(x)$ for $i=1, \ldots, N$. 
The sequence of degrees $\left\{\operatorname{deg}\left(m_{i}(x) r_{i}(x)\right), m_{i}(x) \neq 0\right\}_{i=0, \ldots, N+1}$ is decreasing, while $\left\{\operatorname{deg}\left(m_{i}(x) s_{i}(x)\right), m_{i}(x) \neq 0\right\}_{i=0, \ldots, N+1}$ is increasing. So, if we consider the indexes $k=\min \left\{i: m_{i}(x) \neq 0\right\}$ and $k^{\prime}=\max \left\{i: m_{i}(x) \neq 0\right\}$, then

$$
\kappa(y(x))=\left(\operatorname{deg} r_{k}(x)+\operatorname{deg} m_{k}(x)\right)+\left(\operatorname{deg} s_{k^{\prime}}(x)+\operatorname{deg} m_{k^{\prime}}(x)\right) .
$$

In particular, if we want to minimize this quantity, we may assume that both $m_{k}(x)$ and $m_{k^{\prime}}(x)$ are nonzero elements of $\mathbb{K}$. From $(5.2)$ and Remark 2.2 we deduce that

$$
\begin{aligned}
\operatorname{deg} r_{k}(x) & +\operatorname{deg} s_{k^{\prime}}(x) \\
& =\left(n-\operatorname{deg} q_{1}(x)-\cdots-\operatorname{deg} q_{k}(x)\right)+\left(\operatorname{deg} q_{1}(x)+\cdots+\operatorname{deg} q_{k^{\prime}-1}(x)\right) .
\end{aligned}
$$

This quantity is equal to

$$
\begin{cases}n+\left(\operatorname{deg} q_{k+1}(x)+\cdots+\operatorname{deg} q_{k^{\prime}-1}(x)\right), & \text { if } k^{\prime}>k+1 \\ n, & \text { if } k^{\prime}=k+1 \\ n-\operatorname{deg} q_{k}(x), & \text { if } k^{\prime}=k\end{cases}
$$

From here we deduce in a straightforward manner that the minimum gets reached when $k^{\prime}=k$.

The set defining (5.1) is never empty since $\frac{r_{1}(x)}{s_{1}(x)}=\frac{g(x)}{1}$, the Hermite interpolation polynomial, is always an element of this set. Let $k_{0} \leq N$ be the minimum of (5.1). In particular, $k_{0} \leq \operatorname{deg} g(x)<n$.

To prove that any degree $j \geq n$ is feasible, we proceed as follows: choose any two consecutive indexes $1 \leq k<k+1 \leq N$, and let $\lambda \in \mathbb{K} \backslash\{0\}$ be such that

$$
\frac{r_{k}(x)+\lambda r_{k+1}(x)}{s_{k}(x)+\lambda s_{k+1}(x)}
$$

is irreducible (i.e., the numerator and the denominator do not share any common factor) and the denominator does not vanish at $x_{i}, i=1, \ldots, l$. This can be done as in the proof of Theorem 3.7. Then, due to (5.3), we actually have that the $\kappa$-degree of this function is $n$. For $j>n$, we choose $m_{0}(x)$ a general polynomial of degree $j-\left(n-\operatorname{deg}\left(s_{k}(x)+\lambda s_{k+1}(x)\right)\right)$ such that the fraction

$$
\frac{m_{0}(x) r_{0}(x)+r_{k}(x)+\lambda r_{k+1}(x)}{m_{0}(x) s_{0}(x)+s_{k}(x)+\lambda s_{k+1}(x)}=\frac{m_{0}(x) f(x)+r_{k}(x)+\lambda r_{k+1}(x)}{s_{k}(x)+\lambda s_{k+1}(x)}
$$

is irreducible. Then, it is easy to check that the $\kappa$-degree of this function is equal to $j$, which concludes the proof of the Theorem.

As another application of Theorem 5.2, we obtain a description of the solutions of the rational Hermite interpolation problem (see [6, Theorem 2.6] and [17, Exercise 5.42]). Recall the statement of this problem: For a given $d \in \mathbb{N}, 0 \leq d<n$, decide if there exist, and if so compute, polynomials $a(x), b(x)$ of degrees bounded by $d$ and $n-d-1$ respectively, such that $\frac{a(x)}{b(x)}$ interpolates the data. Observe also that the rational Hermite interpolation problem can be phrased as: Decide if there exist, and if so compute, polynomials $a(x), b(x)$ such that $\frac{a(x)}{b(x)}$ interpolates the data with $\operatorname{deg} a(x) \leq d$ and $\kappa\left(\frac{a(x)}{b(x)}\right) \leq n-1$. Note that this is equivalent, by Proposition 2.1. to deciding if there exist such a coprime pair of polynomials $(a(x), b(x))$. 
Corollary 5.3. For $0 \leq d \leq n-1$, let $1 \leq k \leq N$ be such that

$$
\begin{aligned}
\operatorname{deg} r_{k}(x)=n-\left(\operatorname{deg} q_{1}(x)\right. & \left.+\cdots+\operatorname{deg} q_{k}(x)\right) \leq d \\
& <\operatorname{deg} r_{k-1}(x)=n-\left(\operatorname{deg} q_{1}(x)+\cdots+\operatorname{deg} q_{k-1}(x)\right) .
\end{aligned}
$$

If there is a solution of the Hermite interpolation problem for the integer $d$, then this solution is of the form $\frac{p(x) r_{k}(x)}{p(x) s_{k}(x)}$ for some $p(x) \in \mathbb{K}[x]$. The problem is solvable if and only if $r_{k}(x)$ and $s_{k}(x)$ are coprime.

Example 5.4. For the data in Example 3.11 $n=4, N=3, \operatorname{deg} q_{1}(x)=$ $\operatorname{deg} q_{2}(x)=1, \operatorname{deg} q_{3}(x)=2$. The minimal $\kappa$-degree is 2 and the minimal solution is $\frac{6}{x^{2}-3}$. The Hermite polynomial is the other interpolating rational function of degree less than 4 and has degree 3 . The Hermite interpolation problem has no solution for $d=2$.

Example 5.5. For the data in Example 3.12, $n=6$, the minimal $\kappa$-degree is 4 , and the minimal solutions are $x^{4}-10 x^{2}+10$ and $\frac{4}{x^{4}-2 x^{2}+3}$. There are no other solutions of degree less than 6 . The Hermite interpolation problem has no solution for $d=2$ and $d=3$.

Example 5.6. In the generic case with a data of $n$ pairs the minimal $\kappa$-degree is $n-1$ and $g(x)$ is a minimal solution. The fractions $\frac{r_{i}}{s_{i}}$, for $i=1, \ldots, n$, have degree $n-1$ and are minimal solutions if $r_{i}$ and $s_{i}$ are coprime.

\section{REFERENCES}

[1] A. C. Antoulas and B. D. Q. Anderson, On the scalar rational interpolation problem, $I M A$ J. Math. Control Inform. 3 (1986), no. 2-3, 61-88. https://doi.org/10.1093/imamci/3.2-3.61

[2] A. C. Antoulas, Rational interpolation and the Euclidean algorithm, Linear Algebra Appl. 108 (1988), 157-171. MR 0959703

[3] A. C. Antoulas et al., On the solution of the minimal rational interpolation problem, Linear Algebra Appl. 137/138 (1990), 511-573. MR 1067689

[4] B. Beckermann and G. Labahn, Fraction-free computation of matrix rational interpolants and matrix GCDs, SIAM J. Matrix Anal. Appl. 22 (2000), no. 1, 114-144. MR 1779720

[5] A.-L. Cauchy, Mémoire sur les fonctions alternées et sur les sommes alternées, Exercices d'analyse et de physique mathématique, Tome 2, 151-159, Paris, Bachelier, 1841. https://gallica.bnf.fr/ark:/12148/bpt6k96421826/f161.item

[6] T. Cortadellas Benítez, C. D'Andrea and E. Montoro, The set of unattainable points for the rational Hermite interpolation problem, Linear Algebra Appl. 538 (2018), 116-142. MR 3722831

[7] D. A. Cox, T. W. Sederberg and F. Chen, The moving line ideal basis of planar rational curves, Comput. Aided Geom. Design 15 (1998), no. 8, 803-827. MR 1638732

[8] C. D'Andrea, T. Krick and A. Szanto, Subresultants, Sylvester sums and the rational interpolation problem, J. Symbolic Comput. 68 (2015), part 1, 72-83. MR 3283838

[9] H. Hong, Z. Hough and I. A. Kogan, Algorithm for computing $\mu$-bases of univariate polynomials, J. Symbolic Comput. 80 (2017), part 3, 844-874. MR 3574537

[10] S. W. Kahng, Osculatory interpolation, Math. Comp. 23 (1969), 621-629. MR 0247732. 
[11] A. Lascoux, Symmetric functions and combinatorial operators on polynomials, CBMS Regional Conference Series in Mathematics, 99, American Mathematical Society, Providence, RI, 2003. MR 2017492

[12] A. Predonzan, Su una formula d'interpolazione per le funzioni razionali, Rend. Sem. Mat. Univ. Padova 22 (1953), 417-425. MR 0057937.

[13] M. S. Ravi, Geometric methods in rational interpolation theory, Linear Algebra Appl. 258 (1997), 159-168. MR 1444100

[14] G. Rosenhain, Neue Darstellung der Resultante der Elimination von $z$ aus zwei algebraische Gleichungen $f(z)=0$ und $\varphi(z)=0$ vermittelst der Werthe welche die Functionen $f(z)$ und $\varphi(z)$ für gegebne Werthe von $z$ annehmen, J. Reine Angew. Math. 30 (1846), 157-165. https://doi.org/10.1515/crll.1846.30.157

[15] H. E. Salzer, Note on osculatory rational interpolation, Math. Comp. 16 (1962), 486-491. MR 0149648

[16] J. Tan and Y. Fang, Newton-Thiele's rational interpolants, Numer. Algorithms 24 (2000), no. 1-2, 141-157. MR 1784996 .

[17] J. von zur Gathen and J. Gerhard, Modern computer algebra, third edition, Cambridge University Press, Cambridge, 2013. MR 3087522

[18] L. Wuytack, On the osculatory rational interpolation problem, Math. Comput. 29 (1975), 837-843. MR 0371008

Teresa Cortadellas Benítez

Universitat de Barcelona, Facultat d'Educació. Passeig de la Vall d'Hebron 171, 08035

Barcelona, Spain

terecortadellas@ub.edu

Carlos D'Andrea ${ }^{凶}$

Universitat de Barcelona, Departament de Matemàtiques i Informàtica, Gran Via de les Corts

Catalanes 585, 08007 Barcelona, Spain

cdandrea@ub.edu

$U R L:$ http://www.ub.edu/arcades/cdandrea.html

\section{Eulàlia Montoro}

Universitat de Barcelona, Departament de Matemàtiques i Informàtica, Gran Via de les Corts Catalanes 585, 08007 Barcelona, Spain

eula.montoro@ub.edu

Received: March 30, 2019

Accepted: October 16, 2019 